\title{
Surgical management of metastatic lesions of the proximal femur with pathological fractures using intramedullary nailing or endoprosthetic replacement
}

\author{
ZEPING YU*, YAN XIONG* , RUI SHI, LI MIN, WENLI ZHANG, HONGYUAN LIU, \\ XIANG FANG, CHONGQI TU and HONG DUAN \\ Department of Orthopedics, West China Hospital, Sichuan University, Chengdu, Sichuan 610064, P.R. China
}

Received April 24, 2017; Accepted September 28, 2017

DOI: $10.3892 / \mathrm{mco} .2017 .1503$

\begin{abstract}
Endoprosthetic replacement (EPR) and intramedullary nailing (IMN) are the two most commonly applied surgical methods used to treat proximal metastatic lesions; however, indication of the above procedures remains controversial. The aim of the present study was to evaluate the clinical, functional and oncological outcomes of patients who underwent EPR compared to IMN for the treatment of proximal femur metastases to investigate the surgical indication for patients. The records of patients $(n=88)$ with pathological fractures secondary to metastatic tumors of the proximal femur admitted between January 2005 and December 2014 to West China Hospital, Sichuan University (Chengdu, China) were retrospectively studied. A total of 57 patients were treated with EPR (34 males and 23 females; mean age, 62.5 years) and 31 patients were stabilized with IMN (19 males and 12 females; mean age, 60.2 years). Patients were analyzed regarding surgery time, blood loss, hospital stay, Musculoskeletal Tumor Society (MSTS) score, survival, recurrence and complications. The median follow-up period was 12.9 (range, 3-98) months. The median survival time in EPR was 10.0 months and 7.5 months in IMN. The surgery time was $142.6 \pm 22.7 \mathrm{~min}$ in the EPR group and $98.7 \pm 19.5 \mathrm{~min}$ in the IMN group ( $\mathrm{P}=0.001)$. Significantly less blood loss was observed in the IMN group $(345.2 \pm 66.4 \mathrm{ml})$ than in the EPR group (631.5 $\pm 103.6 \mathrm{ml} ; \mathrm{P}=0.001)$. The median hospital stay in the EPR group was 8 (quartile range, 7-9) days and 5 (quartile range, 5-6) days in the IMN group ( $\mathrm{P}=0.001)$. Local recurrence rate was $10.5 \%$ (6/57) in the EPR group and
\end{abstract}

Correspondence to: Dr Hong Duan, Department of Orthopedics, West China Hospital, Sichuan University, 37 Guo Xue Lane, Chengdu, Sichuan 610064, P.R. China

E-mail: duanhong1970@126.com

*Contributed equally

Key words: metastasis, surgical treatment, endoprosthetic replacement, intramedullary nailing, proximal femur
$25.8 \%(8 / 31)$ in the IMN group ( $\mathrm{P}=0.074)$. The complication rates were $10.5 \%(6 / 57)$ in the EPR group and $29.0 \%(9 / 31)$ in the IMN group ( $\mathrm{P}=0.038)$. MSTS-93 score was higher in IMN compared with EPR at 6 weeks postoperatively $(\mathrm{P}=0.001)$, while the EPR group demonstrated a higher score at 6 months postoperatively $(\mathrm{P}=0.001)$. EPR has the advantage of better functional outcomes and higher life quality in the long term, with lower complication rates in treating metastatic lesions of the proximal femur with pathological fractures. EPR is recommended for patients with relatively good general condition and prognosis. IMN is best indicated when the patient's life expectancy is extremely limited.

\section{Introduction}

Metastatic neoplasms of bone are the most frequently observed malignant destructive bone lesions in adults $(1,2)$ that are commonly encountered by orthopedists. Various malignant tumors have the potential to metastasize to bone, most of which originates from the breast, prostate, lung, kidney and colon (3). Advances in early diagnosis and adjuvant therapies contributes to longer life expectancy of patients with metastatic diseases, combined with an increasing popularity in elderly patients suffering with metastatic bone tumors (2). Proximal femur pathological fractures that occur secondary to the metastases have a devastating effect on patients, inducing intractable pain and inhibition to mobility $(4,5)$. Although non-surgical treatment concerning analgesics, radiotherapy and bisphosphonates may effectively relieve pain, pathological fractures rarely heal (6). In the context of recent metastatic bone tumor therapy, operative interventions are still favorable options for patients with impending or actual fractures (4,7-9). The purpose of treatments is to reduce pain and achieve durable reconstructions that may restore function for the expected life span of the patient $(4,5,10)$.

Surgical modalities are widely regarded as effective, most of which include endoprosthetic reconstruction (EPR) (1,3,7,8,10-13), intramedullary nailing (IMN) and open reduction-internal fixation $(4,5,9,10,14)$. Although these surgical strategies are usually palliative, consensus of the optimal surgical procedure has not been reached. When it comes to metastatic lesions of the proximal femur, surgical management even 
appears to be controversial with Musculoskeletal Tumor Society (MSTS) members in literatures, particularly in intertrochanteric and subtrochanteric regions of the femur (15). Multiple factors regarding the primary tumor, fracture displacement, health status, estimated survival and anatomic region of involvement are of marked importance when choosing appropriate surgical approaches. However, the focus of surgical preferences is generally concentrated on the durability of the implants in previous literatures $(4,5,14)$ and functional outcomes; however, a constant functional follow-up has rarely been analyzed in previous research. The present retrospective study was performed to analyze clinical, functional and oncological outcomes in patients following EPR or IMN in order to investigate the indications for each surgical treatment.

\section{Patients and methods}

Patients. Inclusion criteria for surgery were as follows: i) Metastatic bone tumors with biopsy-proven evidence; ii) radiological findings of impending (Mirels score $\geq 9$ ) or existing pathologic fractures (16); and iii) intractable pain and functional impairment. Exclusion criteria were summarized as follows: Non-surgical procedures, no fracture, close reduction and internal fixation, primary surgery at other hospitals, primary bone tumors, as well as malignancies of lymphoid tissue or blood. Patients in good general condition with major surgical tolerance and better prognosis were treated with EPR. Patients in poor general condition with limited surgical tolerance and poor prognosis were treated with IMN.

A total of 88 patients with impending or displaced pathological proximal femur fractures secondary to metastases received surgery between January 2005 and December 2014 at the West China Hospital, Sichuan University (Chengdu, China). Data on age, sex, type of fracture, surgery time, blood loss, hospital stay, functional and oncological outcomes, as well as postoperative complications, were collected.

A total of 57 patients were treated with EPR (34 males and 23 females; mean age, 62.5 years) and 31 patients were stabilized with IMN (19 males and 12 females; mean age, 60.2 years). Preoperative evaluations included a complete physical examination, blood tests to access general condition, a chest computed tomography (CT) and total-skeleton technetium-99 bone scanning to confirm the existence of other metastasis sites of bone and $\mathrm{CT} / \mathrm{magnetic}$ resonance imaging (MRI) of pelvis and femur to assess the extent of bone and muscle involvements in detail. During surgery, $1 \mathrm{~g}$ tranexamic acid (Chongqing Lummy Pharmaceutical Co., Ltd., Chongqing, China) was administered intravenously to all patients to reduce blood loss.

All procedures performed in the present study involving human participants were in accordance with the ethical standards of the Institutional and National Research Committee and with the 1964 Declaration of Helsinki and its later amendments or comparable ethical standards. Approval for the present study was obtained from the Human and Ethics Committee at West China Hospital of Sichuan University (Chengdu, China).

Adjuvant therapy. All patients enrolled in the present study received adjuvant therapy based on general condition and tumor origins individually. Bisphosphonate zoledronic acid (Chia Tai Tianqing Pharmaceutical Group Co., Ltd., Lianyungang, China) was administered intravenously (4 mg) to all patients once diagnosed to relieve pain, enhance bone density and delay the bone destruction progress (17). Lapatinib [GlaxoSmithKline (China) Investment Co., Ltd., Beijing, China] was used orally for patients with human epidermal growth receptor 2-positive breast cancer if indicated. Tyrosine kinase inhibitor, gefitinib (AstraZeneca Wuxi Trading Co., Ltd., Wuxi, China), was administered orally to patients with non-small cell lung cancer and esophageal cancer if qualified. Chemotherapy and radiotherapy were used in all patients indicated.

\section{Surgical treatments}

EPR group. Surgery proceeded with the patients under general anesthesia in a lateral position and a long posterolateral incision was made, which was extended proximal, distal or in the ilioinguinal area if an extensive soft tissue involvement existed in these areas. The iliotibial band was longitudinally opened to achieve exposure of the attachment of the gluteus maximus muscle at the femur. The gluteus maximus and medius were then detached. The vastus lateralis was reflected distally from its origin and reserved for latter coverage of the prosthesis. Muscles related to the hip joint were detached and the proximal femur was dislocated anterolaterally. Subsequently, femoral osteotomy was performed at $3-5 \mathrm{~cm}$ beyond the farthest point of the lesion, as determined by preoperative MRI. The whole procedure for exposure and tumor removal was completed in normal soft tissue and bone if possible, and hemostasis was required due to the skin incision.

EPR was performed by intramedullary canal reaming, prosthetic implantation and cementing. A capsule was sutured around the head of the prosthesis to form a noose and was reinforced by tenodesis of the pectineus and psoas muscles to the anterior capsule and the external rotators to the posterior capsule, which provided immediate stability and decreased the chance of dislocation. Finally, the abductor mechanism was reconstructed by rotating the vastus lateralis proximally to overlie the prosthesis. The remaining muscles were sutured to the vastus lateralis anteriorly and the hamstrings posteriorly.

IMN group. Surgery was performed on atraction bed in a supine position. A limited incision was created at the tumor site. The vastus lateralis muscle was cut open longitudinally to adequately expose the femoral cortex and a fenestration was made at the lesion site. Reduction of the fractures was conducted and the tumor cavity was devitalized with curettage, alcohol and high-speed burr. The IMN was implanted and adjusted to confirm the proper location and length. Subsequently, the initially placed nail was partially removed, and the tumor cavity was reconstructed with cement. The intramedullary nail was inserted again and locked by an interlocking nail.

Statistical analysis. Continuous data were expressed as the mean \pm standard deviation. Nonparametric variables were expressed as the median and quartile range. Independent samples t-tests were used to analyze the data and nonparametric Mann-Whitney U tests were used for non-normal distribution 
variables regarding hospital stay. Functional outcome was evaluated by the MSTS-93 system (18). Survival curves were calculated using the Kaplan-Meier method, and comparison was analyzed by long-rank test. Pearson's chi-squared test was used to compare recurrence rate and complication rate. Statistical analysis was performed with SPSS v. 20.0 (IBM Corp., Armonk, NY, USA). P<0.05 was considered to indicate a statistically significant difference.

\section{Results}

Clinical and demographic data. In the 57 patients, the lung was the most common primary region of involvement in the EPR group $(28 / 57 ; 49.1 \%)$, followed by the liver $(12 / 57 ; 21.1 \%)$, kidney $(4 / 57 ; 4.0 \%)$ and breast $(4 / 57 ; 4.0 \%)$. Tumor origins in the IMN group indicated that lung $(13 / 31 ; 41.9 \%)$, liver $(8 / 31$; $25.8 \%)$ and colorectal $(4 / 31 ; 12.9 \%)$ involvement predominated. All patients presented with pain and limited mobility. Displaced pathological fractures were present in 37 patients $(64.9 \%)$ in the EPR group and 21 patients $(67.7 \%)$ in the IMN group (Table I). It was observed that blood loss, hospital stay and surgery time were all significantly higher in the EPR group than in the IMN group (all $\mathrm{P}=0.001$ ). No significant difference was observed in any other baseline data (Table I).

Functional and oncological outcomes. All patients demonstrated significantly higher MSTS-93 scores at 6 weeks postoperatively than corresponding preoperative scores $(\mathrm{P}<0.001)$. MSTS-93 score was significantly higher in the IMN group than in the EPR group at 6 weeks post-operation $(\mathrm{P}=0.001)$, while the EPR group demonstrated a significantly higher MSTS-93 score than the IMN group at 6 months post-operation ( $\mathrm{P}=0.001$; Table II). No significant difference was observed between the two groups at 3 months following surgery $(\mathrm{P}=0.152)$. The median survival time with EPR was 10.0 months and 7.5 months with IMN, and there was no significant difference between the two groups ( $\mathrm{P}=0.103$; Fig. 1). In the EPR group, the median survival time was 10.0 months [95\% confidence interval (CI), 8.86-10.94] with a survival rate of $28.7,13.4$ and $10.7 \%$ at 1, 2 and 3 years, respectively. In the IMN group, the median survival time was 7.5 months $(95 \% \mathrm{CI}$, 6.61-8.39) with a survival rate of $19.0,11.4$ and $3.8 \%$ at 1,2 and 3 years, respectively. Local recurrence rate was $10.5 \%(6 / 57)$ in the EPR group and $25.8 \%(8 / 31)$ in the IMN group $(\mathrm{P}=0.074$; Table III). Patients who suffered local recurrence were treated with radiotherapy and no further surgery was conducted. A patient with breast cancer in the present study received allo/autograft prosthesis composite replacement combined with lapatinib and achieved $>8$ years survival with graft-host union (Fig. 2). Furthermore, a patient with lung cancer with poor prognosis received an IMN procedure with gefitinib and demonstrated bone union 18 months postoperatively (Fig. 3).

Complications. The overall complication rate was $17.0 \%$ $(15 / 88)$ in the present study, and $10.5 \%(6 / 57)$ in the EPR group and $29.0 \%(9 / 31)$ in the IMN group $(\mathrm{P}=0.038)$. In the EPR group, 1/57 (1.8\%) patient suffered a systemic complication of deep vein thrombosis in the early post-operative period, which was treated successfully with medical therapy. There was no mortality intra-operatively. Local complications were
Table I. Comparison of clinically related data in patients of the EPR and IMN groups.

\begin{tabular}{|c|c|c|c|}
\hline \multirow[b]{2}{*}{ Characteristic } & \multicolumn{2}{|c|}{ Group } & \multirow[b]{2}{*}{ P-value } \\
\hline & $\operatorname{EPR}(n=57)$ & IMN (n=31) & \\
\hline Age, years & $62.5 \pm 6.2$ & $60.2 \pm 6.6$ & 0.111 \\
\hline Sex & & & 1.000 \\
\hline Male & 34 & 19 & \\
\hline Female & 23 & 12 & \\
\hline Tumor type, n (\%) & & & - \\
\hline Lung & $28(49.1)$ & $13(41.9)$ & \\
\hline Liver & $12(21.1)$ & $8(25.8)$ & \\
\hline Kidney & $4(7.0)$ & $2(6.5)$ & \\
\hline Breast & $4(7.0)$ & $1(3.2)$ & \\
\hline Prostate & $2(3.5)$ & $2(6.5)$ & \\
\hline Colorectal & $3(5.3)$ & $4(12.9)$ & \\
\hline Others & $4(7.0)$ & $1(3.2)$ & \\
\hline Fracture, n (\%) & & & 0.819 \\
\hline Impending & $20(35.1)$ & $10(32.3)$ & \\
\hline Displaced & $37(64.9)$ & $21(67.7)$ & \\
\hline \multicolumn{4}{|l|}{$\begin{array}{l}\text { Type of resection, } \\
\mathrm{n}(\%)\end{array}$} \\
\hline Wide & $57(100.0)$ & $0(0.0)$ & \\
\hline Intralesional & $0(0.0)$ & $31(100.0)$ & \\
\hline Surgery time, $\min$ & $142.6 \pm 22.7$ & $98.7 \pm 19.5$ & 0.001 \\
\hline Blood loss, ml & $631.5 \pm 103.6$ & $345.2 \pm 66.4$ & 0.001 \\
\hline Hospital stay, days ${ }^{a}$ & $8(7,9)$ & $5(5,6)$ & 0.001 \\
\hline
\end{tabular}

${ }^{a}$ Values are expressed as the median and quartile range (P25, P75). EPR, endoprosthetic replacement; IMN, intramedullary nailing.

observed in 5 patients in the EPR group (8.8\%) and 9 patients in the IMN group (29.0\%). A total of 3 patients in the EPR group and 1 patient in the IMN group suffered early superficial wound infection, and were all treated successfully with dressing change and antibiotic therapy. In the IMN group, 3 patients suffered from pneumonia and were treated with aerosol inhalation and anti-infection medication. No deep infections were reported. Dislocation occurred at 3 months postoperatively in a 65-year-old woman in the EPR group $(1.8 \%)$, and a manipulative reduction was performed. The implant-related complication rate was $16.1 \%(5 / 31)$ in the IMN group. A total of 2 patients suffered nonunion at 6 months and 11 months postoperatively, which was treated by revisions to a larger diameter with bone grafts and no further complications occurred. A total of 3 patients suffered an implant breakage and their general condition deteriorated. These patients lost their lives without a second chance for surgery. Pain relief was achieved in all patients following surgery (data not shown).

\section{Discussion}

High weight-bearing and rotational forces make the proximal femur the most frequently involved site of metastases (19). 
Table II. Functional comparisons measured by MSTS-93 score in each group.

\begin{tabular}{lccc}
\hline & \multicolumn{2}{c}{ MSTS-93 score } & Number of patients \\
\cline { 2 - 3 } Time & EPR & IMN & $(57 / 31)$ \\
\hline $\begin{array}{l}\text { Pre-operation } \\
\text { Post-operation }\end{array}$ & $8.14 \pm 2.34$ & $7.68 \pm 2.07$ & $(57 / 31)$ \\
6 weeks & $18.21 \pm 1.87^{\mathrm{a}}$ & $20.68 \pm 2.26^{\mathrm{b}}$ & $(57 / 30)$ \\
3 months & $22.01 \pm 2.82$ & $22.97 \pm 3.09$ & $(41 / 23)$ \\
6 months & $25.39 \pm 2.10$ & $23.17 \pm 2.87$ & 0.001 \\
\hline
\end{tabular}

${ }^{\mathrm{a}} \mathrm{P}<0.001$ vs. preoperative score in EPR group; ${ }^{\mathrm{b}} \mathrm{P}<0.001$ vs. preoperative score in IMN group. MSTS, Musculoskeletal Tumor Society; EPR, endoprosthetic replacement; IMN, intramedullary nailing.

Table III. Oncological outcomes in patients of the EPR and IMN groups.

\begin{tabular}{lccc}
\hline & \multicolumn{2}{c}{ Group } & \\
\cline { 2 - 3 } Variable & EPR & IMN & P-value \\
\hline Survival rate, \% & & & 0.103 \\
1 year & 28.7 & 19 & \\
2 year & 13.4 & 11.4 & \\
3 year & 10.7 & 3.8 & \\
Local recurrence, \% & 10.5 & 25.8 & 0.074 \\
\hline
\end{tabular}

EPR, endoprosthetic replacement; IMN, intramedullary nailing.

High-pace development of diagnosis and adjuvant therapies ameliorate the prognosis of cancer patients, thus contributing to the detection rate of skeletal-related metastatic events. Femoral metastases regularly induce chronic intractable pain and functional impairment, particularly in patients with pathological fracture (1). Patients who have received non-surgical treatments with pathological fractures inevitably have a long bed rest time, which commonly leads to complications that deteriorate the prognosis, including hypostatic pneumonia, bedsore, urinary infection and deep vein thrombosis. As pathological fractures induced by metastases rarely heal (6), surgical treatments are necessary in such cases. Various therapeutic strategies have been applied for treating metastases located at the proximal femur, and the basic treatment goals should include pain relief and functional restoration by achieving local tumor control and immediate mechanical stability $(4,5,10)$.

In the present study, it was observed that all clinical-related data collected concerning surgery time, blood loss and hospital stay were higher in the EPR group. This result is in accordance with a study by Algan and Horowitz (20). In the present study, an en-bloc resection of the tumor was performed, whose margins were located in normal tissues, thus a complete hemostasis was able to be achieved before the implantation of the endoprosthesis. Despite this, more blood loss was observed in the EPR group than in the IMN group, as anticipated prior to

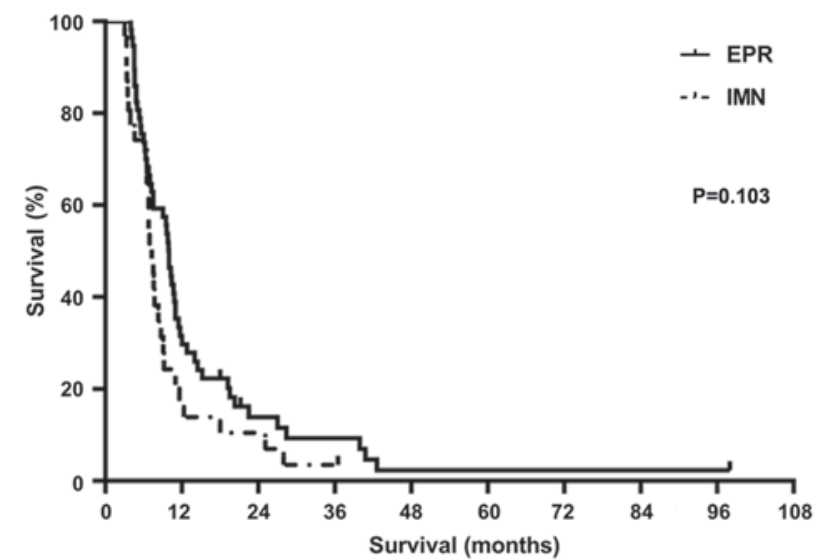

Figure 1. Kaplan-Meier survivorship curve with $95 \%$ confidence intervals. No significant difference was observed between patients who received EPR or IMN. EPR, endoprosthetic replacement; IMN, intramedullary nailing.

surgery. A long incision was used to get the surgical exposure as large as possible, which contributed to a complete resection combined with increased blood loss. On the contrary, surgical steps in the IMN group were not as complicated as EPR. Minimal incision, a precisely located surgical area, and lesion curettage without reconstruction made IMN a minor trauma procedure that was less time consuming than EPR.

Radical excision of tumor was achieved in the EPR group combined with resection of the gluteus maximus and medius, vastus lateralis and capsule of the hip joint. Even though the muscles and capsule resected were reconstructed, early hip function was not as good in the EPR group as it was in the IMN group. Regarding the IMN group, the muscle group around the hip were all reserved, which provided good early active mobilization postoperatively compared with the EPR group. These surgical characteristics distinguished some patients with extremely limited survival time, because shorter recovery and good early limb function are of vital importance to them. Therefore, EPR may be a more invasive approach indicated for patients with major surgical tolerance and good general condition. IMN is best indicated for patients with relatively poor general condition.

Pathological fractures secondary to metastatic lesions generally indicate the terminal stage of malignant tumor 

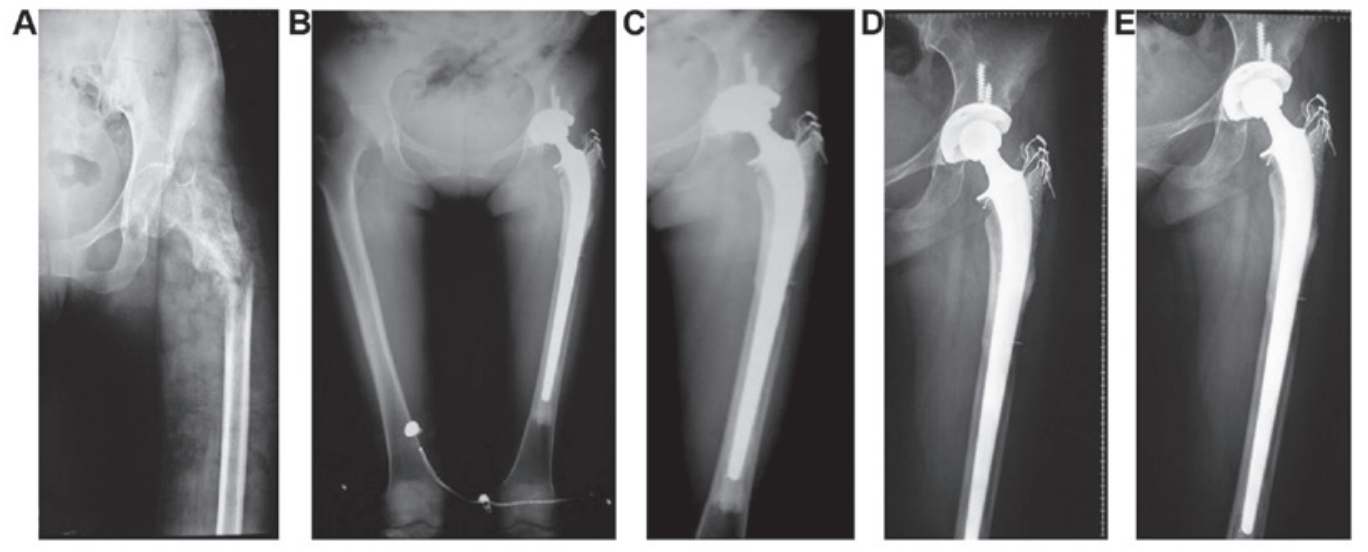

Figure 2. X-ray images of a 47-year-old patient with a left proximal femur pathological fracture secondary to breast cancer treated with APC replacement. (A) Preoperative X-ray images. (B) and (C) X-ray imaging following APC replacement at 36-month clinical follow-up. Graft-host union was observed. (D) and (E) X-ray imaging at a 96-month follow-up. No dislocation, recurrence or implant failure were observed. APC, allo/autograft prosthesis composite.
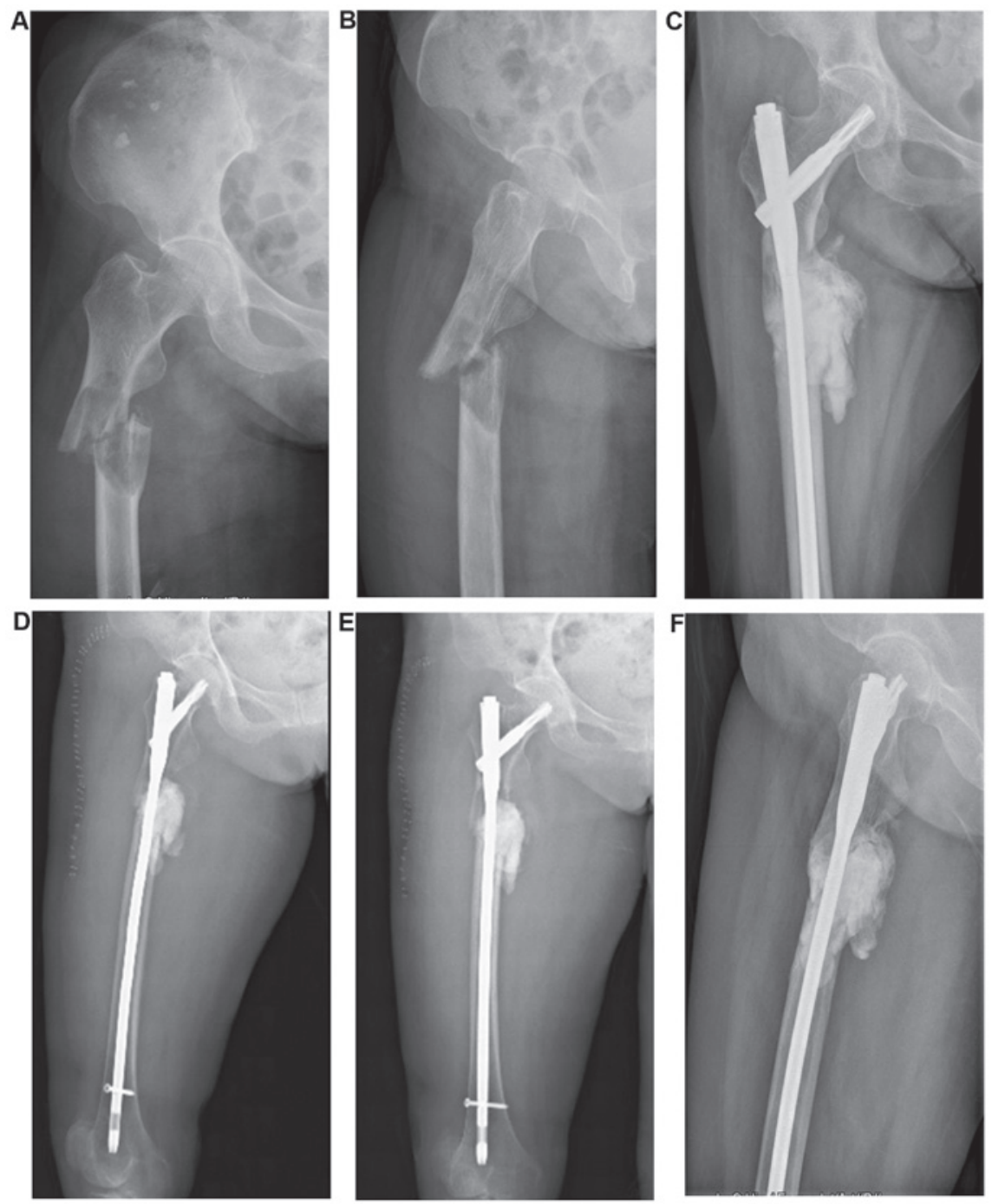

Figure 3. X-ray images of a 57-year-old patient with a right proximal femur pathological fracture secondary to lung cancer treated with intramedullary nailing. (A) and (B) Preoperative X-ray images. (C) and (D) X-ray imaging at a 3-month follow-up. (E and F) Bone union was observed at 18 months postoperatively. No local recurrence or implant failure were observed.

progression, which suggests that estimated survival time and recovery should be taken into consideration when making choices about surgical treatments $(21,22)$. Patients with prox- imal metastatic fractures in the present study demonstrated a survival rate of 28.7, 13.4 and $10.7 \%$ at 1,2 and 3 years, respectively, in the EPR group. Patients in the IMN group 
Table IV. Oncological outcomes in peer-reviewed literature for the surgical treatment of pathological femur fractures.

\begin{tabular}{|c|c|c|c|c|c|c|}
\hline \multirow[b]{2}{*}{ Authors, year } & \multirow[b]{2}{*}{$\begin{array}{l}\text { Level of } \\
\text { evidence }\end{array}$} & \multirow[b]{2}{*}{$\begin{array}{l}\text { Surgical } \\
\text { strategy }\end{array}$} & \multirow[b]{2}{*}{ Anatomic site(s) } & \multicolumn{2}{|c|}{ Results/outcomes } & \multirow[b]{2}{*}{ (Refs.) } \\
\hline & & & & $\begin{array}{c}\text { Median } \\
\text { survival, months }\end{array}$ & $\begin{array}{c}\text { Survival rate } \\
\text { (1 year/2 year } \\
13 \text { year), } \%\end{array}$ & \\
\hline $\begin{array}{l}\text { Sarahrudi et al, } \\
2009\end{array}$ & III & $\begin{array}{l}\operatorname{IMN}(n=94) \\
\operatorname{EPR}(n=23) \\
\text { ORIF }(n=22)\end{array}$ & Femur & 3.7 & $17.0 / 6.0^{\mathrm{a}}$ & (7) \\
\hline $\begin{array}{l}\text { Sarahrudi et al, } \\
2006\end{array}$ & III & $\begin{array}{l}\operatorname{IMN}(n=53) \\
\operatorname{EPR}(n=18) \\
\text { ORIF }(n=13)\end{array}$ & Femur; humerus & 3.2 & $16.2 / 7.0 / 4.5$ & (12) \\
\hline $\begin{array}{l}\text { Wedin and Bauer, } \\
2005\end{array}$ & III & $\begin{array}{l}\operatorname{IMN}(n=24) \\
\operatorname{EPR}(n=109) \\
\text { ORIF }(n=13)\end{array}$ & Femur & $\begin{array}{c}15 \text { months } \\
\text { with reoperation; } \\
3 \text { months without } \\
\text { reoperation }\end{array}$ & $30.0 / 10.0 / 7.0$ & (10) \\
\hline Selek et al, 2008 & IV & $\operatorname{EPR}(n=45)$ & Femur & 11.5 & $27.2^{\mathrm{b}}$ & (8) \\
\hline Present study & III & $\begin{array}{l}\text { EPR }(n=57) \\
\operatorname{IMN}(n=31)\end{array}$ & Proximal femur & 9.0 & 25.3/12.8/8.1 & - \\
\hline
\end{tabular}

${ }^{\mathrm{a}} 1$-year/2-year survival rate; ${ }^{\mathrm{b}} 1$-year survival rate. EPR, endoprosthetic replacement; IMN, intramedullary nailing; ORIF, open reduction and internal fixation.

demonstrated a survival rate of $19.0,11.4$ and $3.8 \%$ at 1,2 and 3 years, respectively. Various survival outcomes have been reported in other studies; however, poor survival has predominated. A study by Sarahrudi et al (12) conducted extensive research concerning 88 patients surgically treated for metastatic lesions and reported a survival rate of $16.2 \%$ at 1 year, $7 \%$ at 2 years and $4.5 \%$ at 3 years postoperatively in 2006 . A long-term retrospective study involving 142 patients treated with EPR and reconstruction nailing for 146 metastatic lesions conducted by Wedin and Bauer (10) demonstrated a survival rate of $30 \%$ at 1 year, $10 \%$ at 2 years and $7 \%$ at 3 years postoperatively. The survival outcomes observed in the present study are comparable to these results (Table IV). However, a study by Harvey et al (5) reported a markedly higher 1-year survival rate of 51\%, 2-year survival rate of $29 \%$ and 5-year survival rate of $11 \%$ in a study of 158 patients with proximal femur metastases. Different baseline information and selection bias in each study may lead to varying tumor compositions, cancer stage and health status, which may partially explain such large differences between study results. In the present study, lung cancer along with liver cancer accounted for 70.2 and $67.7 \%$ in the EPR and IMN group, respectively, as an independent predictor contributing to reduced survival (23), which deteriorated the overall prognosis in the present study.

In the present study, no significant difference was observed between the overall survival rates of the two groups, even though the two types of resection were completely different, which may indicate that surgery does not increase survival time. The aim of surgery is to achieve local tumor control, increase limb function, improve quality of life, decrease prognosis-worsening factors, including skeletal-related events and bed rest-related complications, so as to improve survival indirectly (24). The combination of surgical treatment and adjuvant therapy is of great importance and the choice of adjuvant therapy depends on the tumor type, generation condition and patients' affordability $(13,25,26)$. A patient with breast cancer in the present study who received allo/autograft prosthesis composite replacement combined with lapatinib achieved $>8$ years survival with graft-host union. Furthermore, a patient with lung cancer with poor prognosis received an IMN procedure with gefitinib and demonstrated bone union 18 months postoperatively. These cases indicated that a potentially longer survival and acceptable function may be achieved in the terminal stage of cancer in patients if an appropriate surgical approach and comprehensive treatment are utilized.

However, surgical decision-making, most of which is based on surgeons' clinical judgment (27), should be guided under a uniform scoring system. A prognostic factors and scoring system used to estimate expected survival time for patients with skeletal metastasis was proposed by Katagiri et al (23), which considers survival, the site of the primary lesion, the performance status, the presence of visceral or cerebral metastases, any previous chemotherapy and multiple skeletal metastases as five important prognostic factors. In the present study, all patients received survival estimations; however, some patients with a lower score demonstrated longer survival than those patients with higher scores. This finding indicates that it is difficult to accurately estimate survival time.

Constant estimations of functional outcomes are rarely observed in published literatures. The postoperative functional outcomes in the present study were evaluated at 6 weeks, 3 and 6 months in order to define if the functional change and differences in each group would affect further choices of surgical methods. In the present study, the MSTS-93 scores in the two groups were significantly increased at 6 weeks post-operation compared with pre-operation. However, the 
MSTS scores in EPR and IMN differed with time. Significant differences were observed at 6 weeks and 6 months after surgery. The IMN group demonstrated better scores at 6 weeks post-operation, while the EPR group demonstrated better scores at 6 months post-operation. No significant difference was observed at 3 months following surgery. The changes of the functional outcome based on time change may be used as a reference to influence decision-making, which conforms with the present study design of determining surgical indication for different patients. Therefore, for the patients who required a higher level of functional outcomes and higher quality of life with a longer life span, EPR was the first choice, if possible. On the contrary, patients with limited expected survival time should receive procedures that have shorter recovery times.

The time of improvement in patients with metastatic disease is critically important. Improvements in pain control and function occurred as early as 6 weeks postoperatively in the present study. Any potential surgical benefits must occur as soon as possible. The present findings support the rule of a 6-week life expectancy to consider surgery (22). It is possible that patients with a shorter life expectancy may also benefit from surgery (22).

The overall complication rate was $17.0 \%(15 / 88)$ in the present study, with complication rates of $29.0 \%$ (9/31) in the IMN group and $10.5 \%(6 / 57)$ in the EPR group. Implant failure rates regarding proximal femur pathological fractures vary vastly in different literatures, ranging from $0-23 \%(5,12,28,29)$. A study by Manoso et al (28) reported the most favorable results that no infections, dislocations or reoperations were observed in a 6-year retrospective study with 13 cancer patients with proximal femur metastasis treated with proximal femur replacement. The present study demonstrated a higher implant breakage rate of $9.67 \%(3 / 31)$ in the IMN group than in the EPR group (0\%). Lower failure rate in EPR was also observed by Harvey et al (5), who observed a $0 \%$ failure rate in EPR vs. $11 \%$ in IMN. Union and nail breakage were the primary cause of implant failure in the present study, and lower implant-related complications in EPR have been reported in various literatures $(4,5,10)$. Thoroughly intramedullary curettage of malignant tumors is an indispensable process in IMN group causing large bone defect, in which leads to high chance of bone union. In the present study, patients were generally elderly with osteoporosis, and when such patients receive this palliative surgery they should be treated with radiology therapy postoperatively in case of local progression. Long stem nails function as a load-sharing component for the healing of the fracture, whereas endoprosthesis does not require the healing of bone (5). These limitations of IMN make union and breakage a difficult problem to overcome, particularly in patients with an estimated longer survival $(10,30)$. A dislocation rate of $1.8 \%(1 / 57)$ in the EPR group the present study was observed, which is comparable to previously reported results, ranging from $0-13.8 \%(5,8,10,28)$. Reserved muscle was used in the present study to fully cover the endoprosthesis and enhance the capsule to enable reliable reconstruction.

The present study was limited by its retrospective design, which degraded its level of evidence to III. Secondly, we recommended EPR as an optimal choice for some patients with resectable involvements that had a better prognosis, which may have meant that the IMN group contained more patients with more advanced-stage cancer. Though all efforts were made to eliminate selection bias, this kind of selection bias concerning specific patients exists extensively and is difficult to eliminate. The reason is that the surgical treatment regarding metastatic bone lesions should be applied individually $(22,31)$. EPR is a radical resection, however it has excessive cost compared with IMN. Surgical decisions are not only based on medical characters, but also health economics (14), particularly in China. Patients with extremely limited lifespan will not benefit from major surgery and trauma as such procedures require longer recovery. Nevertheless, no significant difference was observed concerning survival and recurrence between the two groups. However, all patients in our institution received surgery from the same senior surgeon in a single medical center and were all followed up by the same medical team. Furthermore, the type of resection was controlled to be accordant with surgical procedures and we controlled the confounding factors among baseline information in both groups to guarantee the accuracy of the difference between the two most commonly used surgical methods. The present study demonstrated that favorable outcomes may be achieved in EPR and IMN when patients are appropriately selected.

In conclusion, patients with proximal metastatic lesions of proximal femur benefit from surgical management. As surgery will not improve survival directly, shorter recovery and improved limb function with lower complications are of vital importance. EPR is indicated for patients with relatively better general condition and prognosis. In the present study, the EPR group achieved better effects of functional outcome in the long term compared with IMN for treating proximal femur metastasis. However, lower complication rates are of crucial importance for patients with longer life expectancy. Minor surgical trauma, quicker recovery and better early limb function in the IMN group make it the best indicated for patients in poor general condition with substantially limited life expectancy.

\section{References}

1. Camnasio F, Scotti C, Peretti GM, Fontana F and Fraschini G: Prosthetic joint replacement for long bone metastases: Analysis of 154 cases. Arch Orthop Trauma Surg 128: 787-793, 2008.

2. Hage WD, Aboulafia AJ and Aboulafia DM: Incidence, location and diagnostic evaluation of metastatic bone disease. Orthop Clin North Am 31: 515-528, 2000.

3. Clarke HD, Damron TA and Sim FH: Head and neck replacement endoprosthesis for pathologic proximal femoral lesions. Clin Orthop Relat Res: 210-217, 1998

4. Steensma M, Boland PJ, Morris CD, Athanasian E and Healey JH: Endoprosthetic treatment is more durable for pathologic proximal femur fractures. Clin Orthop Relat Res 470: 920-926, 2012.

5. Harvey N, Ahlmann ER, Allison DC, Wang L and Menendez LR: Endoprostheses last longer than intramedullary devices in proximal femur metastases. Clin Orthop Relat Res 470: 684-691, 2012.

6. Gainor BJ and Buchert P: Fracture healing in metastatic bone disease. Clin Orthop Relat Res: 297-302, 1983.

7. Sarahrudi K, Greitbauer M, Platzer P, Hausmann JT, Heinz T and Vecsei V: Surgical treatment of metastatic fractures of the femur: A retrospective analysis of 142 patients. J Trauma 66: 1158-1163, 2009.

8. Selek H, Basarir K, Yildiz Y and Sağlik Y: Cemented endoprosthetic replacement for metastatic bone disease in the proximal femur. J Arthroplasty 23: 112-117, 2008

9. Gao H, Liu Z, Wang B and Guo A: Clinical and functional comparison of endoprosthetic replacement with intramedullary nailing for treating proximal femur metastasis. Chin J Cancer Res 28: 209-214, 2016. 
10. Wedin R and Bauer H: Surgical treatment of skeletal metastatic lesions of the proximal femur: Endoprosthesis or reconstruction nail? J Bone Joint Surg Br 87: 1653-1657, 2005.

11. Lane JM, Sculco TP and Zolan S: Treatment of pathological fractures of the hip by endoprosthetic replacement. J Bone Joint Surg Am 62: 954-959, 1980.

12. Sarahrudi K, Hora K, Heinz T, Millington S and Vécsei V: Treatment results of pathological fractures of the long bones: A retrospective analysis of 88 patients. Int Orthop 30: 519-524, 2006.

13. Xing Z, Moon BS, Satcher RL, Lin PP and Lewis VO: A long femoral stem is not always required in hip arthroplasty for patients with proximal femur metastases. Clin Orthop Relat Res 471: 1622-1627, 2013.

14. Tanaka T, Imanishi J, Charoenlap $\mathrm{C}$ and Choong PF: Intramedullary nailing has sufficient durability for metastatic femoral fractures. World J Surg Oncol 14: 80, 2016.

15. Steensma M and Healey JH: Trends in the surgical treatment of pathologic proximal femur fractures among musculoskeletal tumor society members. Clin Orthop Relat Res 471: 2000-2006, 2013.

16. Mirels $\mathrm{H}$ : Metastatic disease in long bones: A proposed scoring system for diagnosing impending pathologic fractures. Clin Orthop Relat Res: 256-264, 1989.

17. Van Acker HH, Anguille S, Willemen Y, Smits EL and Van Tendeloo VF: Bisphosphonates for cancer treatment: Mechanisms of action and lessons from clinical trials. Pharmacol Ther 158: 24-40, 2016.

18. Enneking WF, Dunham W, Gebhardt MC, Malawar M and Pritchard DJ: A system for the functional evaluation of reconstructive procedures after surgical treatment of tumors of the musculoskeletal system. Clin Orthop Relat Res: 241-246, 1993.

19. Jacofsky DJ and Haidukewych GJ: Management of pathologic fractures of the proximal femur: State of the art. J Orthop Trauma 18: 459-469, 2004

20. Algan SM and Horowitz SM: Surgical treatment of pathologic hip lesions in patients with metastatic disease. Clin Orthop Relat Res: 223-231, 1996.
21. Damron TA: Management of metastatic carcinoma to the hip and proximal femur. In Menendez LR (ed). Orthopaedic Knowledge Update: Musculoskeletal Tumors. Rosemont, IL, American Academy of Orthopaedic Surgeons 363-371, 2002.

22. Talbot M, Turcotte RE, Isler M, Normandin D, Iannuzzi D and Downer P: Function and health status in surgically treated bone metastases. Clin Orthop Relat Res 438: 215-220, 2005.

23. Katagiri H, Takahashi M, Wakai K, Sugiura H, Kataoka T and Nakanishi K: Prognostic factors and a scoring system for patients with skeletal metastasis. J Bone Joint Surg Br 87: 698-703, 2005.

24. Tsuya A, Kurata T, Tamura K and Fukuoka M: Skeletal metastases in non-small cell lung cancer: A retrospective study. Lung Cancer 57: 229-232, 2007.

25. Body JJ: New developments for treatment and prevention of bone metastases. Curr Opin Oncol 23: 338-342, 2011.

26. Coluzzi F, Di Bussolo E, Mandatori I and Mattia C: Bone metastatic disease: Taking aim at new therapeutic targets. Curr Med Chem 18: 3093-3115, 2011.

27. Schneiderbauer MM, von Knoch M, Schleck CD, Harmsen WS, Sim FH and Scully SP: Patient survival after hip arthroplasty for metastatic disease of the hip. J Bone Joint Surg Am 86-A: 1684-1689, 2004.

28. Manoso MW, Frassica DA, Lietman ES and Frassica FJ: Proximal femoral replacement for metastatic bone disease. Orthopedics 30: 384-388, 2007.

29. Yazawa Y, Frassica FJ, Chao EY, Pritchard DJ, Sim FH and Shives TC: Metastatic bone disease. A study of the surgical treatment of 166 pathologic humeral and femoral fractures. Clin Orthop Relat Res: 213-219, 1990

30. Dijstra S, Wiggers T, van Geel BN and Boxma H: Impending and actual pathological fractures in patients with bone metastases of the long bones. A retrospective study of 233 surgically treated fractures. Eur J Surg 160: 535-542, 1994.

31. Narazaki DK, de Alverga Neto CC, Baptista AM, Caiero MT and de Camargo OP: Prognostic factors in pathologic fractures secondary to metastatic tumors. Clinics (Sao Paulo) 61: 313-320, 2006. 